\title{
Multilayered transmissive disc
}

\author{
Jean-Claude Lehureau, Joseph Colineau \\ Thales Research and Technology, \\ Domaine de Corbeville, 91404 Orsay, France
}

Optical storage is the leading technology for removable storage media. In order to follow the roadmap of other computer components, an annual capacity growth of $30 \%$ to $60 \%$ is needed. In the past, this was reached by decreasing the wavelength and increasing the numerical aperture; however, the next generation of DVR will approach practical limits with a numerical aperture of 0.85 and a wavelength of 405 nanometers. In order to reach capacities in the range of terabytes, volume recording is needed. We show that minor changes in the architecture of optical pick up can allow multilayer recording of a few hundred layers. New media are needed but solutions are foreseen for Read Only and Write Once media. Magneto-optic and Phase-change rewritable media offer a poor modulation to absorption ratio; therefore, multilayer rewritable media is still a challenge.

Key words: optical disc, optical storage, focused recording, push pull, multilayer, inverse saturable absorber, non-linear organics

\section{Introduction}

According to Moore's law, the complexity of an integrated circuit is multiplied by a factor of 4 every three years; during the last twenty years, the level of integration of solid state processors and memories have increased of four orders of magnitude.

Mass storage subsystem must follow the pace. For example, during the same lap of time, magnetic storage density evolved from less than $100 \mathrm{Mbits} / \mathrm{square}$ inch to $100 \mathrm{Gbits} / \mathrm{square}$ inch. Optical storage started with a huge advance when it appeared; but since, it has only evolved from a 1 Gbyte 12 " disc to a 5 Gbyte 5" DVD. Optical storage will remain the solution for removable and distribution media only if it can make a quantum leap of capacity in the near future.

Surface density of information is related to diffraction limit; i.e. to square of readout wavelength divided by the square of numerical aperture.

$$
\mathrm{D}=\mathrm{K} \cdot \mathrm{NA}^{2} / \lambda^{2}
$$

Where $\mathrm{K}$ is the number of bits per Airy spot. $\mathrm{K}$ is a quality factor related to the perfection of components and the complexity of signal processing.

$$
\begin{aligned}
\text { Typically: } & K=2 \text { for } C D, \\
K=5 & \text { for } D V D .
\end{aligned}
$$

The factors of progress from CD to DVD have been:

1- $\quad$ shorter wavelength : X 1.4

2- $\quad$ higher numerical aperture : X 2
3- better signal processing : X 2.5

The future standards ${ }^{1) 2}$ will use a blue laser ( X 2.8) and even higher numerical aperture $(\mathrm{X} 1.7)$ but there is no room for future improvement of the signal processing $(\mathrm{K} \sim 7)$.

In a further future, wavelength cannot be decreased far below $405 \mathrm{~nm}$ because of limitations of source and transparency of media and optics; numerical aperture cannot be increased toward unity without loosing the main advantage of optical media : the high flying height of optics that allows working even in a dusty environment.

Volume recording is the only way to increase significantly the density. A reasonable thickness of media could allow stacking more than 1000 layers of information if the transparency is sufficient. Holography has been proposed for many years but media and components are not available for mass production of a cheap drive.

We show that focused recording can increase storage capacity by at least two orders of magnitude. It takes advantage of existing components. Solutions are proposed for efficient energy budget and simple pick up architecture.

\section{Readout Energy Budget}

Single mode laser diode provides typically $30 \mathrm{~mW}$ recording power. This is true for IR, red and even blue lasers. Assuming a $30 \%$ collecting efficiency of the optics, recording power on the disc is typically $10 \mathrm{~mW}$. Because reading power must remain far below the recording threshold, it should be in the $1 \mathrm{~mW}$ range.

Reflective multilayered recording is already part of DVD standard. The main drawback of this structure is that readout light power on the detectors strongly decreases when the number of layers increases. Figure 1 shows the reflectivity needed for a given ratio of light return. It shows that 11 layer disc could be theoretically stacked if the light efficiency has to be $5 \%$ and that 17 layer disc is possible if the detectors can cope with a reflectivity of $3 \%$. Practically, the limit is given by the noise of the detectors. For example at its introduction, CD-RW, which has a reflectivity of only $20 \%$, required to modify the standard of the $\mathrm{CD}$ reader. As wavelength decreases and data rate increases it will be a real challenge to cope with efficiency below $5 \%$. No more than ten layers have been demonstrated in lab environment and the market feasibility of more than three layers is questionable. 


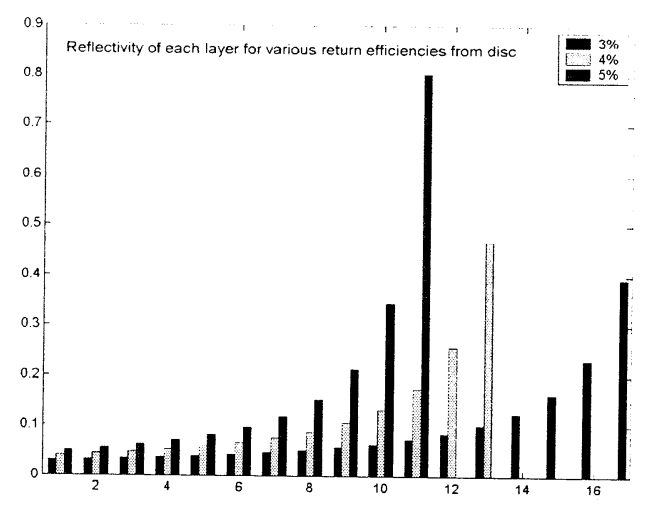

Fig 1 Reflectivity of each layer for a given light return ratio

If the sensitivity of the detectors is increased, the light return ratio of each layer can be decreased and the number of layers increases almost proportionally. At Nyquist rate, detector's noise equivalent power (NEP) is typically 1000 photons/sample and is mostly due to the first stage of electronic amplifier.

Optical heterodyning is a well-known solution that can increase the detectivity up to $\mathrm{S} / \mathrm{N}=6 \mathrm{~dB}$ per photon. Magneto-optic is a practical application of optical heterodyning. Kerr rotation can be seen as the generation of a light signal polarized perpendicular to the polarization of the incident beam. In a magneto-optic pick up, the electric field associated with Kerr rotation is added to a part of the non-rotated component reflected by the disc and projected on a polarization analyzer.

Figure 2 shows typical energy budget of magneto-optic readout. Most of laser output $(1 \mathrm{~mW})$ is transmitted through the partially polarizing beamsplitter to the disc, whereas $10 \%$ only of the returning beam $(100 \mu \mathrm{W})$ is reflected towards the polarization detector. The power associated with the magneto-optic signal is proportional to the square of the Kerr rotation and is in fact less than 10 nanowatts but it modulates the reflected power by a few microwatts.

The main difference between reading a magneto-optic layer and a semi-reflective layer is that while magnetooptic reflects both modulated and DC component, the semi-reflective layer reflects the modulated component and transmits the main light power. Adding laser light in the same spatial mode could amplify the reflected component on the detector plane; this however would require a sophisticated tracking system and four phase synchronous detection in order to process interference between a fix beam inside the pick up with the faint reflection from the moving disc.

On the contrary when reading a transmissive layer, it is quite simple to exploit the interference patterns that appear inside the transmitted beam. Furthermore, because it can be made of pure dielectric mediums, the absorption is null and the phase shift is proportional to the depth of the physical modulation of the interface of two dielectric mediums.

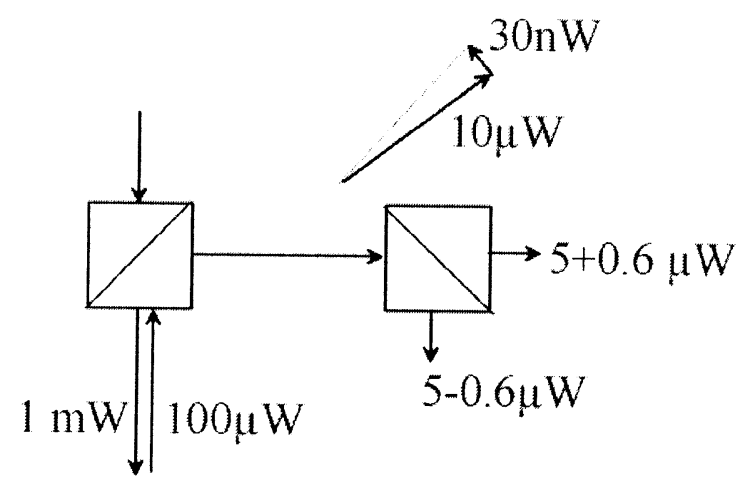

Fig 2 Energy budget of magneto-optic readout

When the phase shift is small, the wavefront transmitted through the information layer can be analyzed into DC component and diffracted component. This diffracted field interferes in phase or in opposition with the DC component. When the information layer is not at focus, the far field interference pattern looks like a casted shadow of the information layer. As the information layer approaches to the focus plane, the spatial frequency of the casted shadow decreases. When focused, the casted shadow of a pit edge will resume in a forward/backwards shift of the far field power distribution. This latter case is well known as Push Pull signal for tracking or readout ${ }^{3)}$. Push Pull can be easily explained as a deflection of the beam when the information dot is entering the focus spot; The staircase form of the wavefront can be approximated by a tilted wavefront which corresponds to a shift of the far field. Push Pull signal amplitude is proportional to the phase shift of the information dot whereas Central Aperture signal is proportional to its square. Figure 3 shows the computed far field modulation when a $\lambda / 30$ pits is crossing the waist of the beam.

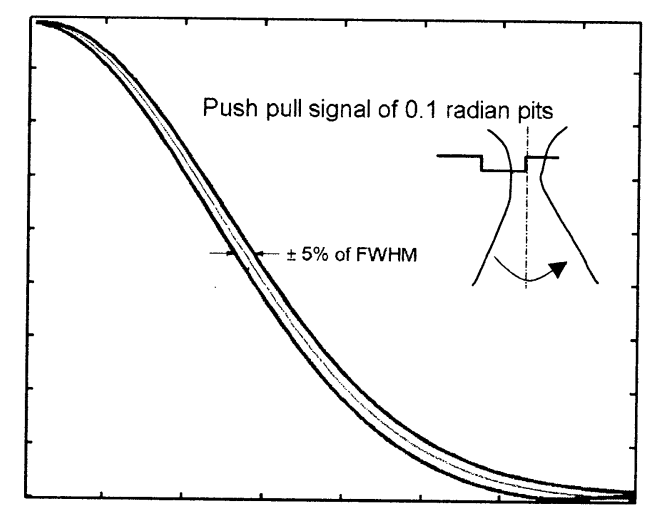

Fig 3 Computed Far Field deflection of a gaussian beam 
If $\alpha$ is the phase shift between rays passing through or outside the dot, the transmission through the information layer generates a deflection of the beam:

$$
\mathrm{D}= \pm 2 \alpha / \mathrm{T} \quad(\mathrm{T} \text { is the spot size) }
$$

Compared to the NA, the relative deflection is:

$$
\text { Dr }= \pm \alpha / \pi
$$

The relative modulation on each half detector is twice that value:

$$
M_{\text {peak/DC }}= \pm 2 \alpha / \pi
$$

The loss due to diffusion is equal to the variance of phase therefore the transmittance of undiffracted beam is:

$$
\mathrm{T}=1-\alpha^{2 / 4}
$$

For example a phase shift $\alpha=0.1$ gives a relative signal of $7 \% 0$-peak/DC while the transmittance of each layer is better than $99.75 \%$.

\section{Structure of a Read Only Disk}

A phase shift $\alpha=0.1$ radian corresponds to a difference in optical path:

$$
\Delta \mathrm{n} \mathrm{e}=5 \mathrm{~nm}
$$

Typical CD mastering process generates $100 \mathrm{~nm}$ deep dots. A transmissive information layer can be formed at the interface of two mediums with index difference:

$$
\Delta \mathrm{n}=0.005
$$

This variation of index of refraction can be easily obtained by changing the nature of polymer or by adding plasticizer to a same polymer. Of course, 100 interfaces cannot be generated by continuously increasing the index of refraction. But two mediums with different indices can be alternatively stacked. The readout signal polarity will simply be inverted if the index change is inverted; the tracking signals are not even inverted.

For production, a photopolymerization process similar to the one used for two layer DVD can be used. Since no metallization is needed between layers, the process can be repeated at fast rate.

Although the readout of each layer is basically a transmissive readout process, it would be difficult on a real product to split and align the pick up into an upper and lower part; therefore we plan to metallize the last layer which incidentally can work as a conventional reflective disk. This last feature could be of interest for backward compatibility.

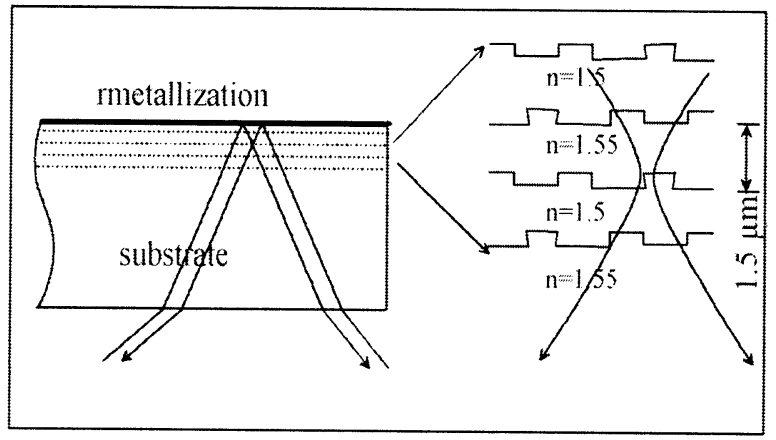

Figure 4 Structure of Read Only disc

\section{Structure of a Write Once Disc}

The volume to be heated around a write once layer is defined by the heat diffusion depth ${ }^{4)} \mathrm{d}$, the width of the spot $w$ and the speed of the media $v$.

$$
\begin{array}{r}
\mathrm{V}=2 \mathrm{~d} w \mathrm{wt} \quad \text { with } \mathrm{d}^{2}=\tau(\mathrm{K} / \mathrm{c}) \\
\text { and } \mathrm{w}=0.5 \lambda / \mathrm{NA}
\end{array}
$$

Future standard will use blue laser $\lambda=400 \mathrm{~nm}$ with $\mathrm{NA}=0.8$ writing a spot in $\tau=10 \mathrm{~ns}$ at $\mathrm{v}=25 \mathrm{~m} / \mathrm{s}$; with these typical conditions the heat will diffuse in polymer medium at $\mathrm{d}=20 \mathrm{~nm}$ below and above the absorbing layer.

The volume to be heated is $0.2510^{-6} \mathrm{~cm}^{3}$ per second and it requires some $200 \mu \mathrm{W}$ to reach a degradation temperature of $400^{\circ} \mathrm{C}$.

If 100 layers have to be stacked, the absorption of each layer must be kept significantly well below $1 \%$ in order to keep some light for readout. The writing beam needs to be one order of magnitude above the typical value of commercial recorders.

$\mathrm{CD}-\mathrm{R}$ discs are recorded by thermal bleaching of an organic dye. It is well known that these dyes have nonlinear properties under high illumination. The molecules are excited by photons from the ground state to a transitory singlet state $\mathrm{S} 1$; thermal relaxation enables a transition to a more stable triplet state $\mathrm{T} 1$.

Generally, inverse saturable absorption occurs due to the higher cross section of the triplet state. There is however quite a few dyes that have a sufficient lifetime of the S1 state for recording at $100 \mathrm{Mbits} / \mathrm{s}$. The cross section of the transition from S1 to a higher S2 state can be very high and the relaxation from $S$ ? to $S 1$ very fast. Porphyrins are good candidates for this type of non linearity.

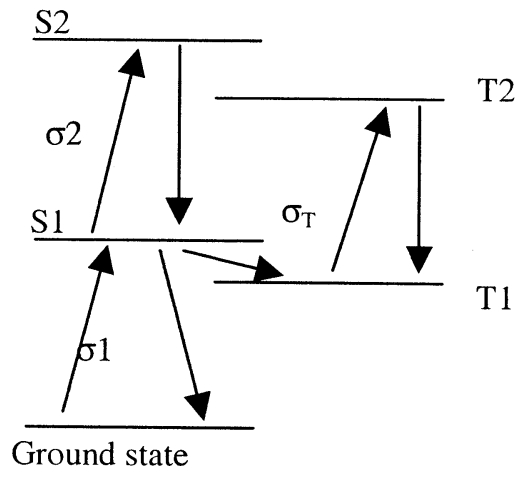

Figure 5 Transition towards singlet and triplet states 
Table 1 Cross section and transition time of some dyes ${ }^{5}$

\begin{tabular}{|c|c|c|c|c|}
\hline Molecules & $\sigma 2 / \sigma_{1}$ & $\tau_{\mathrm{f}}(\mathrm{ns})$ & $\tau_{\mathrm{ST}}(\mathrm{ns})$ & $\mathrm{h} \nu / \sigma_{1} \mathrm{~J} / \mathrm{cm}^{2}$ \\
\hline Si-phtalocyanine & 12 & 4.5 & 0.35 & 0.2 \\
\hline Sn-phtalocyanine & 10 & 2 & 0.62 & 0.25 \\
\hline Zn-TMS-porphyrine & 34 & 7.4 & 34 & 0.15 \\
\hline $\mathrm{Cu}$-TMS-porphyrine & 7 & 7 & 7 & 0.03 \\
\hline
\end{tabular}

The threshold of non-linear effect is related to the cross section of the ground state. The energy given in table 1 is to compare to the light intensity at the focus of a $10 \mathrm{~mW}$ writing beam.

$$
\mathrm{E}=10^{-2} /(0.5 \lambda / \mathrm{NA})^{2}=16 \mathrm{MW} / \mathrm{cm}^{2}
$$

It shows that the increase of absorption of the layer on which beam is focused may occurs within a few nanoseconds. According to the ratio of cross sections $\sigma 2 / \sigma 1$ the absorption may increase by one order of magnitude. Defocused layer on the contrary will not be submitted to the threshold light density within the relaxation time $\tau_{\mathrm{f}}$ and will remain transparent.

Figure 6 shows a recorded pattern on a phtalocyanine disc using $\lambda=350 \mathrm{~nm} 10 \mathrm{~ns}$ pulse at $20 \mathrm{MW} / \mathrm{cm}^{2}$; the absorption of the dye was only $5 \%$ at this wavelength.

The thickness of the heated volume is only $40 \mathrm{~nm}$ according to the heat diffusion equation; it is unlikely that thermal degradation of polymer generates an index variation $\Delta \mathrm{n}=0.12$ as needed for a phase shift of $5 \mathrm{~nm}$ for readout. Kramers Kronig equations ${ }^{6)}$ relates the phase response of a filter to its amplitude. If the laser frequency is on the slope of an absorption edge of the dye, it is reasonable to expect a phase modulation typically 10 times bigger than the amplitude modulation; therefore, a $0.5 \%$ absorption can generate a 50 milliradian phase modulation which is adequate for push pull readout.

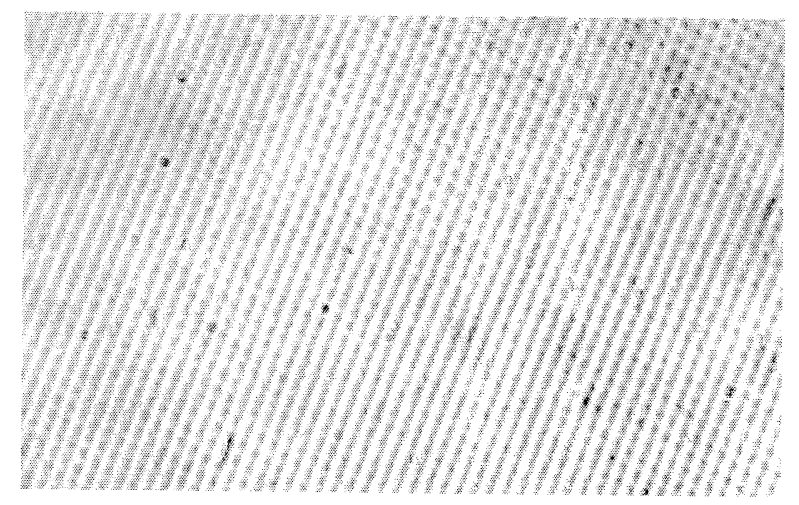

Figure 6 Recorded pattern on phtalocyanine disc

\section{Radial and Vertical crosstalk}

Central Aperture readout requires a phase reference to a land; on the contrary, Push Pull has self reference along the track; therefore no land is needed to separates the tracks Optimally, the adjacent tracks are centered on the first zero crossing of the Airy spot. The optimum track pitch is related to the numerical aperture of the lens:

$$
\mathrm{Tw}=\lambda / 2 \mathrm{~N} . \mathrm{A} .=0.25 \mu \mathrm{m} \text { for blue DVD }
$$

A $30 \%$ gain in radial density can be expected from the absence of land.

Vertical crosstalk can be understood as each layer casting its shadow on the detector plane; the spatial frequency of this modulation is proportional to the spatial frequency of recorded pattern multiplied by the defocusing distance. A common way to get rid of higher spatial frequency is to filter the beam at a focus point as in confocal microscopy. Unfortunately, refocusing the transmitted beam adds complexity to the pick up architecture and the positioning of the filtering pinhole is not compatible with mechanical stability of the pick up. It is well known that multiplying a distribution is equivalent to a convolution in the Fourier domain. Therefore, confocal imaging can be replaced by a convolutive filtering in the far field. This is performed by profiling the sensitivity of the detectors in the tangential direction. Theoretically, crosstalk is reduced to zero if the spatial frequency of the projected pattern is higher than the width of the Fourier transform of the detector sensitivity function. The spectrum of run length limited signal can extend typically from $1 / 4$ to $3 / 4$ of the optical cutoff frequency:

For N.A. $=0.8, \lambda=0.4 \mu \mathrm{m}$ :

Optical cut off frequency:

$$
\Lambda=\lambda / 2 . \text { N.A. }=0.25 \mu \mathrm{m}
$$

Longest recorded wavelength

$$
4 \Lambda=1 \mu \mathrm{m}
$$

Projected pattern at defocus

$$
4 \Lambda /(d / n)
$$

Main component of detector sensitivity function $1 / 2$ N.A.

Typical spacing between layers

$$
4 \Lambda /(\mathrm{d} / \mathrm{n})>2 \text { N.A. } \rightarrow \mathrm{d}>1 \mu \mathrm{m}
$$

Simulations show that a physical spacing of $1.5 \mu \mathrm{m}$ is quite adequate for a reduced crosstalk and good servo signals.

\section{Simulation and PU architecture}

The simulation of the readout process is conducted using a beam propagation method.

1- A light distribution is generated in the pupil
plane
$2-\quad$ Focused spot is computed by Fourier
transform
$3-\quad$ Beam is back propagated at the first layer

3- Beam is back propagated at the first layer 
4- Electric field is computed after phase modulation by the layer

5- Angular spectral density is computed and propagated at the next layer

6- Electric field is computed after phase modulation by second layer

7- After $\sim 80$ layers, beam is propagated in the far field

8- Output signal are computed by integration of power distribution.

Figure 7 shows the result of readout simulation while increasing the pit depth. It confirms that the signal grows linearly up to a value of $10 \mathrm{~nm}$. Above that value distortion increases without increasing the signal.

Ray tracing simulation has been performed in order to identify the key parameter of a pick up. Volume recording implies a variation in cover thickness; therefore, the thickness of the recorded volume must be kept to a minimum. The spherical aberration due to cover thickness variation grows drastically when the numerical aperture is larger than $\mathrm{NA}=0.8$; but on an other hand, volume density varies as the fourth power of NA. Therefore a good compromise should settle for $\mathrm{NA} \cong 0.8$ on a $200 \mu \mathrm{m}$ thick volume. In the latter case a small correction of spherical aberration is needed.

Figure 8 shows the ray tracing simulation for upper and lower layers. All reflected beams have the same cross section at the object focus of the lens. The weighted beam separator must be placed near this plane and is preferably a diffractive component.

\section{Conclusion}

The structure of the multilayer disc allows a surface density up to $3200 \mathrm{bits} / \mu \mathrm{m}^{2}$, which corresponds to:

$$
\begin{aligned}
& \text { Trackwidth }=0.25 \mu \mathrm{m} \\
& \text { Bit length }=0.125 \mu \mathrm{m} \\
& 100 \text { layers at } 1.5 \mu \mathrm{m} \text { spacing }
\end{aligned}
$$

This allows a promising roadmap up to 4 Tbyte $12 \mathrm{~cm}$ disc. Even if it seems futuristic, one has to consider that 4 Terabytes is just 2 generations above the future standard

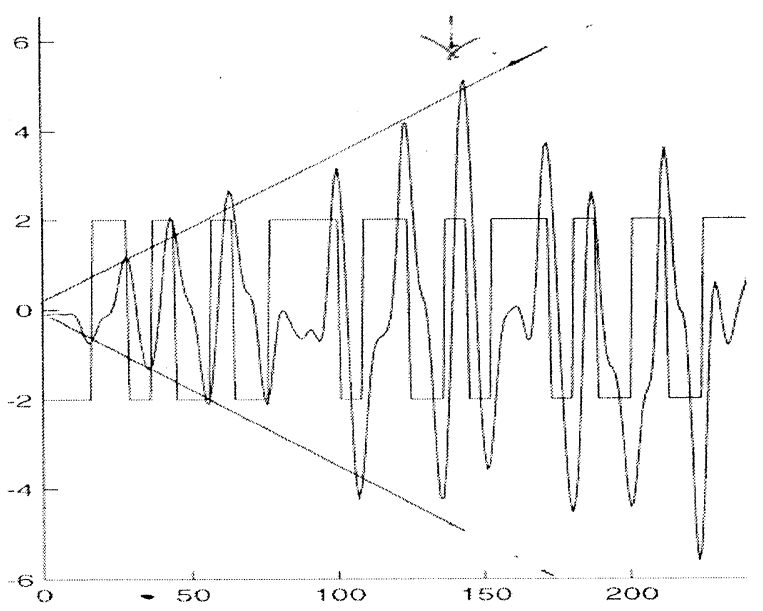

Figure $7 \mathrm{RF}$ signal as a function of pit's phase shift (mRd) of "blue ray disc".

Such a new generation of product definitely needs to foresee a solution for recordable disc. Organic dye recording is a sound solution. In order to solve the problem of rewritable one must find a rewritable media able to modulate the readout beam with low absorption. At present the figure of merit (modulation/absorption) of phase change and metallic magneto-optic layer cannot allow more than 2 or 3 layers to be stacked. Oxide magneto-optic layer could reach the target if performances that are obtained in the infrared could be extended to the near UV.

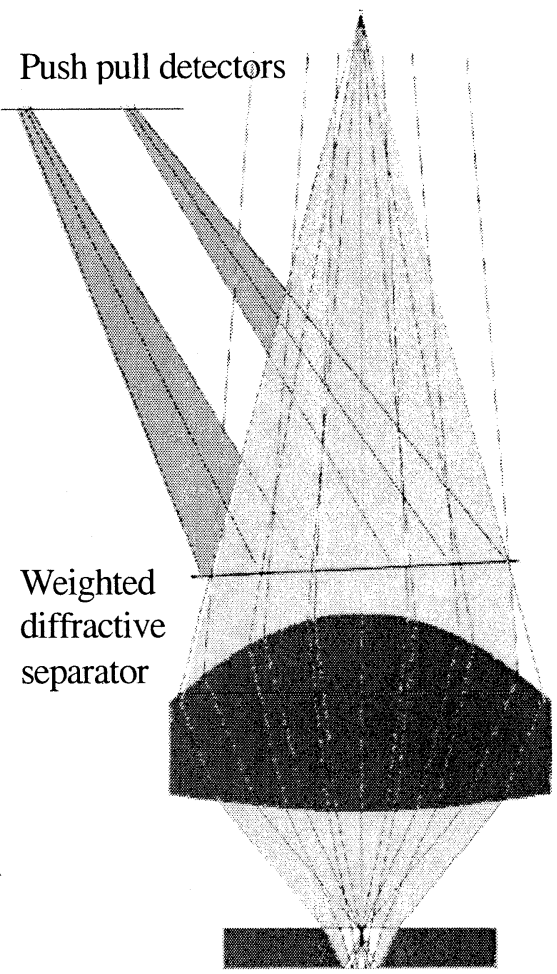

Figure 8 Ray tracing simulation of the pick up

\section{References}

1) K.Shep,B. Stek, R. van Woudenberg. M. Blüm, S. Kobayashi T. Narahara, T. Yamagami, H. Ogawa: Tech Digest ISOM 2000 p210.

2) K. Kurokawa, T. Yamasaki, T. Nakao, K. Mano, K. Yasuda, S. Takagawa, M. Nakamura Tech. Digest ODS 2001.

3) G. Bouwhuis, J. Braat, A. Huisjer, J. Pasman, G. van Rosmalen, K. Schouhamer Immink: Principles of optical disc systems Adam Hilger Ltd (1985).

4) Cornet J. SPIE proc, 420, 57 (1983).

5) J. D. Swalen, F. Kajzar : Non linear absorption in optical limiting Int. Symp. On Optical Power Limiting (2000).

6) R. de L. Kronig, H.A. Kramers Atti Congr. Intern. Fisici, Como 2, 545 (1927) 